\title{
Dunkl-type generalization of the second kind beta operators via $(p, q)$-calculus
}

\author{
Md. Nasiruzzaman', Abdullah Alotaibi² and M. Mursaleen ${ }^{2,3,4^{*}}$ (1)
}

\section{"Correspondence:}

mursaleenm@gmail.com

${ }^{2}$ Operator Theory and Applications

Research Group, Department of

Mathematics, Faculty of Science,

King Abdulaziz University, Jeddah,

Saudi Arabia

${ }^{3}$ Department of Medical Research,

China Medical University Hospital, China Medical University (Taiwan),

Taichung, Taiwan

Full list of author information is

available at the end of the article

\begin{abstract}
The main purpose of this research article is to construct a Dunkl extension of $(p, q)$-variant of Szász-Beta operators of the second kind by applying a new parameter. We obtain Korovkin-type approximation theorems, local approximations, and weighted approximations. Further, we study the rate of convergence by using the modulus of continuity, Lipschitz class and Peetre's K-functionals.
\end{abstract}

MSC: Primary 41A25; 41A36; secondary 33C45

Keywords: $(p, q)$-integers; Dunkl exponential function; $(p, q)$-beta operators; Szász-Beta operators; Weighted spaces

\section{Introduction and preliminaries}

The $q$-analogues of Bernstein operators were independently given by Lupaş [25] and Phillips [42]. Consequently, Mursaleen et al. [33] applied the $(p, q)$-integers and studied the approximation properties of Bernstein operators. Recently, a Dunkl-type generalization of Szász operators [47] via post-quantum calculus was studied by Alotaibi et al. [10]. For more details and research motivation in Dunkl-type generalizations, we mention here some research articles $[13,27,34,35,37-39,45,46]$. Let $[s]_{p, q}$ be the $(p, q)$-integer defined as

$$
\begin{aligned}
& {[s]_{p, q}=p^{s-1}+q p^{s-3}+\cdots+q^{s-1}= \begin{cases}\frac{p^{s}-q^{s}}{p-q} & (p \neq q \neq 1), \\
\frac{1-q^{s}}{1-q} & (p=1), \\
s & (p=q=1),\end{cases} } \\
& (a u+b v)_{p, q}^{s}:=\sum_{\ell=0}^{s} p^{\frac{(s-\ell)(s-\ell-1)}{2}} q^{\frac{\ell(\ell-1)}{2}}\left[\begin{array}{l}
s \\
\ell
\end{array}\right]_{p, q} a^{s-\ell} b^{\ell} u^{s-\ell} v^{\ell}, \\
& (1-u)_{p, q}^{s}=(1-u)(p-q u)\left(p^{2}-q^{2} u\right) \cdots\left(p^{s-1}-q^{s-1} u\right), \\
& (u-y)_{p, q}^{s}= \begin{cases}\prod_{j=0}^{s-1}\left(p^{j} u-q^{j} y\right) & \text { if } s \in \mathbb{N}, \\
1 & \text { if } s=0 .\end{cases}
\end{aligned}
$$

(c) The Author(s) 2020. This article is licensed under a Creative Commons Attribution 4.0 International License, which permits use sharing, adaptation, distribution and reproduction in any medium or format, as long as you give appropriate credit to the original author(s) and the source, provide a link to the Creative Commons licence, and indicate if changes were made. The images or other third party material in this article are included in the article's Creative Commons licence, unless indicated otherwise in a credit line to the material. If material is not included in the article's Creative Commons licence and your intended use is not permitted by statutory regulation or exceeds the permitted use, you will need to obtain permission directly from the copyright holder. To view a copy of this licence, visit http://creativecommons.org/licenses/by/4.0/. 
The $(p, q)$-power basis is explained as

$$
(u \oplus v)_{p, q}^{s}=(u+v)(p u+q v)\left(p^{2} u+q^{2} v\right) \cdots\left(p^{s-1} u+q^{s-1} v\right) .
$$

Furthermore, the $(p, q)$-analogues of the exponential function are defined by

$$
e_{p, q}(u)=\sum_{\ell=0}^{\infty} p^{\frac{\ell(\ell-1)}{2}} \frac{u^{\ell}}{[\ell]_{p, q} !}, \quad E_{p, q}(u)=\sum_{\ell=0}^{\infty} q^{\frac{\ell(\ell-1)}{2}} \frac{u^{\ell}}{[\ell]_{p, q} !}
$$

Moreover, the $(p, q)$-Dunkl analogue of the exponential function is defined by

$$
\begin{aligned}
& e_{\tau, p, q}(u)=\sum_{\ell=0}^{\infty} p^{\frac{\ell(\ell-1)}{2}} \frac{u^{\ell}}{\gamma_{\tau, p, q}(\ell)}, \\
& \gamma_{\tau, p, q}(\ell) \\
& =\frac{\prod_{i=0}^{\left[\frac{\ell+1}{2}\right]-1} p^{2 \tau(-1)^{i+1}+1}\left(\left(p^{2}\right)^{i} p^{2 \tau+1}-\left(q^{2}\right)^{i} q^{2 \tau+1}\right) \prod_{j=0}^{\left[\frac{\ell}{2}\right]-1} p^{2 \tau(-1)^{j}+1}\left(\left(p^{2}\right)^{j} p^{2}-\left(q^{2}\right)^{j} q^{2}\right)}{(p-q)^{\ell}} .
\end{aligned}
$$

And a recursion identity is defined as

$$
\gamma_{\tau, p, q}(\ell+1)=\frac{p^{2 \tau(-1)^{\ell+1}+1}\left(p^{2 \tau \theta_{\ell+1}+\ell+1}-q^{2 \tau \theta_{\ell+1}+\ell+1}\right)}{(p-q)} \gamma_{\tau, p, q}(\ell),
$$

where

$$
\theta_{\ell}= \begin{cases}0 & \text { for } \ell=2 m, m=0,1,2, \ldots \\ 1 & \text { for } \ell=2 m+1, m=0,1,2, \ldots\end{cases}
$$

For $m=0,1,2, \ldots s$, the number $\left[\frac{m}{2}\right]$ denotes the greatest integer function evaluated at $m / 2$.

In our demonstration, we let $u \geq 0$ and $C[0, \infty)$ be the class of all continuous functions on $[0, \infty)$. Recent investigation in $[10,38]$ defined the $(p, q)$-Dunkl analogue of Szász operators by

$$
D_{s, p, q}(f ; u)=\frac{1}{e_{\tau, p, q}\left([s]_{p, q} u\right)} \sum_{\ell=0}^{\infty} \frac{\left([s]_{p, q} u\right)^{\ell}}{\gamma_{\tau, p, q}(\ell)} p^{\frac{\ell(\ell-1)}{2}} f\left(\frac{p^{\ell+2 \tau \theta_{\ell}}-q^{\ell+2 \tau \theta_{\ell}}}{p^{\ell-1}\left(p^{s}-q^{s}\right)}\right) .
$$

\section{Operators and basic estimates}

In this section we construct a class of $(p, q)$-variant of Szász-Beta operators of the second kind generated by an exponential function via Dunkl generalization in Definition 2.1. Such operators are a generalized version of the operators studied in [7, 22, 28, 29, 31, 36, 45].

Definition 2.1 Let $f \in C_{\zeta}[0, \infty)=\left\{f(t): f(t)=O\left(t^{\zeta}\right), t \rightarrow \infty, f \in C[0, \infty)\right\}$ and consider $u \geq 0, \zeta>s$, and $s \in \mathbb{N}$. Then for all $0<q<p \leq 1, \tau>-\frac{1}{2}$, and $\theta_{\ell}$ given by (1.5), we define

$$
\mathcal{P}_{s, p, q}^{\tau}(f ; u)=\sum_{\ell=0}^{\infty} \mathcal{Q}_{s, p, q}(u) \frac{1}{\mathcal{B}_{p, q}\left(\ell+2 \tau \theta_{\ell}+1, s\right)} \int_{0}^{\infty} \frac{t^{\ell+2 \tau \theta_{\ell}}}{(1 \oplus p t)_{p, q}^{\ell+2 \tau \theta_{\ell}+s+1}} f(t) \mathrm{d}_{p, q} t,
$$


where

$$
\mathcal{Q}_{s, p, q}(u)=\frac{1}{e_{\tau, p, q}\left([s]_{p, q} u\right)} \frac{\left([s]_{p, q} u\right)^{\ell}}{\gamma_{\tau, p, q}(\ell)} p^{\frac{\ell(\ell-1)}{2}}
$$

and $\mathcal{B}_{p, q}\left(\ell+2 \tau \theta_{\ell}+1, s\right)$ is the Beta function of the second kind in post-quantum calculus defined by

$$
\mathcal{B}_{p, q}(\alpha, \beta)=\int_{0}^{\infty} \frac{t^{\alpha-1}}{(1 \oplus p t)_{p, q}^{\alpha+\beta}} \mathrm{d}_{p, q} t, \quad \alpha, \beta \in \mathbb{N}
$$

where a formula for the $(p, q)$-Beta function is given by

$$
\mathcal{B}_{p, q}(\alpha, \beta)=\frac{[\alpha-1]_{p, q}}{p^{\alpha-1}[\beta]_{p, q}} \mathcal{B}_{p, q}(\alpha-1, \beta+1), \quad \alpha, \beta \in \mathbb{N} .
$$

Moreover, to obtain the basic estimates here, we use the following relations:

$$
\begin{aligned}
& {\left[\ell+1+2 \tau \theta_{\ell}\right]_{p, q}=q\left[\ell+2 \tau \theta_{\ell}\right]_{p, q}+p^{\ell+2 \tau \theta_{\ell}},} \\
& {\left[\ell+2+2 \tau \theta_{\ell}\right]_{p, q}=q^{2}\left[\ell+2 \tau \theta_{\ell}\right]_{p, q}+(p+q) p^{\ell+2 \tau \theta_{\ell}} .}
\end{aligned}
$$

For more related results on $(p, q)$-analogues, we refer to $[1-6,8,9,11,14-21,26,30,43$, $44,48]$ and also see $[12,32,40]$, for example, if $p=1$, the operators $\mathcal{P}_{s, p, q}^{\tau}$ reduce to those considered recently (see [45]). We have the following inequalities.

Lemma 2.2 Let $f(t)=1, t, t^{2}$. Then the operators $\mathcal{P}_{s, p, q}^{\tau}(\cdot ; \cdot)$ defined by (2.1) satisfy $\mathcal{P}_{s, p, q}^{\tau}(1 ; u)=1$, and the following inequalities hold:

$$
\mathcal{P}_{s, p, q}^{\tau}(f ; u) \leq \begin{cases}\frac{[s]_{p, q}}{[s-1]_{p, q}} u+\frac{1}{[s-1]_{p, q}} \quad \text { for } f(t)=t, \\ \frac{[s]_{p, q}^{2}}{[s-1]_{p, q}[s-2]_{p, q}} u^{2}+\frac{[s]_{p, q}}{[s-1]_{p, q}[s-2]_{p, q}}\left(1+[2]_{p, q}+[1+2 \tau]_{p, q}\right) u \\ \quad+\frac{[2]_{p, q}}{[s-1]_{p, q}[s-2]_{p, q}} \quad \text { for } f(t)=t^{2},\end{cases}
$$

and

$$
\mathcal{P}_{s, p, q}^{\tau}(f ; u) \geq\left\{\begin{array}{l}
\frac{q[s]_{p, q}}{[s-1]_{p, q}} u+\frac{1}{[s-1]_{p, q}} \quad \text { for } f(t)=t, \\
\frac{\left.q^{3}[s]_{p, q}^{2}\right]}{[s-1]_{p, q}[s-2]_{p, q}} u^{2} \\
\quad+\frac{q[s]_{p, q}}{[s-1]_{p, q}[s-2]_{p, q}}\left(q+[2]_{p, q}+q^{2+2 \tau}[1-2 \tau]_{p, q} \frac{e_{\tau, p, q}\left(\frac{q}{p}[s]_{p, q} u\right)}{e_{\tau, p, q}\left([s]_{p, q} u\right)}\right) u \\
\quad+\frac{[2]_{p, q}}{[s-1]_{p, q}[s-2]_{p, q}} \quad \text { for } f(t)=t^{2} .
\end{array}\right.
$$

Proof To prove the results of this lemma, we use (2.2)-(2.5). Take $f(t)=1$. Then

$$
\begin{aligned}
\mathcal{P}_{s, p, q}^{\tau}(1 ; u) & =\sum_{\ell=0}^{\infty} \mathcal{Q}_{s, p, q}(u) \frac{1}{\mathcal{B}_{p, q}\left(\ell+2 \tau \theta_{\ell}+1, s\right)} \int_{0}^{\infty} \frac{t^{\ell+2 \tau \theta_{\ell}}}{(1 \oplus p t)_{p, q}^{\ell+2 \tau \theta_{\ell}+s+1}} \mathrm{~d}_{p, q} t \\
& =\sum_{\ell=0}^{\infty} \mathcal{Q}_{s, p, q}(u) \frac{\mathcal{B}_{p, q}\left(\ell+2 \tau \theta_{\ell}+1, s\right)}{\mathcal{B}_{p, q}\left(\ell+2 \tau \theta_{\ell}+1, s\right)}=1
\end{aligned}
$$


If $f(t)=t$, then

$$
\begin{aligned}
\mathcal{P}_{s, p, q}^{\tau}(t ; u)= & \sum_{\ell=0}^{\infty} \mathcal{Q}_{s, p, q}(u) \frac{1}{\mathcal{B}_{p, q}\left(\ell+2 \tau \theta_{\ell}+1, s\right)} \int_{0}^{\infty} \frac{t^{\ell+2 \tau \theta_{\ell}+1}}{(1 \oplus p t)_{p, q}^{\ell+2 \tau \theta_{\ell}+s+1}} \mathrm{~d}_{p, q} t \\
= & \sum_{\ell=0}^{\infty} \mathcal{Q}_{s, p, q}(u) \frac{\mathcal{B}_{p, q}\left(\ell+2 \tau \theta_{\ell}+2, s-1\right)}{\mathcal{B}_{p, q}\left(\ell+2 \tau \theta_{\ell}+1, s\right)} \\
= & \frac{q}{[s-1]_{p, q}} \sum_{\ell=0}^{\infty} \mathcal{Q}_{s, p, q}(u) \frac{1}{p^{\ell+2 \tau \theta_{\ell}+1}}\left[\ell+2 \tau \theta_{\ell}\right]_{p, q}+\frac{1}{p[s-1]_{p, q}} \\
= & \frac{1}{p[s-1]_{p, q}}+\frac{q[s]_{p, q}}{p^{2}[s-1]_{p, q}} \sum_{\ell=0}^{\infty} \mathcal{Q}_{s, p, q}(u)\left(\frac{p^{2 \ell+2 \tau \theta_{2 \ell}}-q^{2 \ell+2 \tau \theta_{2 \ell}}}{p^{2 \ell-1}\left(p^{s}-q^{s}\right)}\right) \\
& +\frac{q[s]_{p, q}}{p^{2+2 \tau}[s-1]_{p, q}} \sum_{\ell=0}^{\infty} \mathcal{Q}_{s, p, q}(u)\left(\frac{p^{2 \ell+1+2 \tau \theta_{2 \ell+1}}-q^{2 \ell+1+2 \tau \theta_{2 \ell+1}}}{p^{2 \ell}\left(p^{s}-q^{s}\right)}\right) .
\end{aligned}
$$

Clearly, we have

$$
\begin{aligned}
\mathcal{P}_{s, p, q}^{\tau}(t ; u) & \geq \frac{1}{[s-1]_{p, q}}+\frac{q[s]_{p, q}}{[s-1]_{p, q}} \sum_{\ell=0}^{\infty} \mathcal{Q}_{s, p, q}(u)\left(\frac{p^{\ell+2 \tau \theta_{\ell}}-q^{\ell+2 \tau \theta_{\ell}}}{p^{\ell-1}\left(p^{s}-q^{s}\right)}\right) \\
& =\frac{1}{[s-1]_{p, q}}+\frac{q[s]_{p, q}}{[s-1]_{p, q}} D_{s, p, q}(t ; u) \\
& =\frac{1}{[s-1]_{p, q}}+\frac{q[s]_{p, q}}{[s-1]_{p, q}} u
\end{aligned}
$$

and

$$
\mathcal{P}_{s, p, q}^{\ell, \tau}(t ; u) \leq \frac{1}{[s-1]_{p, q}}+\frac{[s]_{p, q}}{[s-1]_{p, q}} u
$$

Similarly, for $f(t)=t^{2}$, we have

$$
\begin{aligned}
\mathcal{P}_{s, p, q}^{\tau}\left(t^{2} ; u\right)= & \sum_{\ell=0}^{\infty} \mathcal{Q}_{s, p, q}(u) \frac{1}{\mathcal{B}_{p, q}\left(\ell+2 \tau \theta_{\ell}+1, s\right)} \int_{0}^{\infty} \frac{t^{\ell+2 \tau \theta_{\ell}+2}}{(1 \oplus p t)_{p, q}^{\ell+2 \tau \theta_{\ell}+s+1}} \mathrm{~d}_{p, q} t \\
= & \sum_{\ell=0}^{\infty} \mathcal{Q}_{s, p, q}(u) \frac{\mathcal{B}_{p, q}\left(\ell+2 \tau \theta_{\ell}+3, s-2\right)}{\mathcal{B}_{p, q}\left(\ell+2 \tau \theta_{\ell}+1, s\right)} \\
= & \sum_{\ell=0}^{\infty} \mathcal{Q}_{s, p, q}(u) \frac{\mathcal{B}_{p, q}\left(\ell+2 \tau \theta_{\ell}+3, s-2\right)}{\mathcal{B}_{p, q}\left(\ell+2 \tau \theta_{\ell}+1, s\right)} \\
= & \frac{1}{[s-1]_{p, q}[s-2]_{p, q}} \\
& \times \sum_{\ell=0}^{\infty} \mathcal{Q}_{s, p, q}(u) \frac{1}{p^{3+2 \ell+4 \tau \theta_{\ell}+1}}\left[\ell+2 \tau \theta_{\ell}+1\right]_{p, q}\left[\ell+2 \tau \theta_{\ell}+2\right]_{p, q} \\
= & \frac{q^{3}[s]_{p, q}^{2}}{[s-1]_{p, q}[s-2]_{p, q}} \sum_{\ell=0}^{\infty} \mathcal{Q}_{s, p, q}(u) \frac{1}{p^{5+4 \tau \theta_{\ell}}}\left(\frac{p^{\ell+2 \tau \theta_{\ell}}-q^{\ell+2 \tau \theta_{\ell}}}{p^{\ell-1}\left(p^{s}-q^{s}\right)}\right)^{2}
\end{aligned}
$$




$$
\begin{aligned}
& +\frac{q(p+2 q)[s]_{p, q}}{[s-1]_{p, q}[s-2]_{p, q}} \sum_{\ell=0}^{\infty} \mathcal{Q}_{s, p, q}(u) \frac{1}{p^{4+2 \tau \theta_{\ell}}}\left(\frac{p^{\ell+2 \tau \theta_{\ell}}-q^{\ell+2 \tau \theta_{\ell}}}{p^{\ell-1}\left(p^{s}-q^{s}\right)}\right) \\
& +\frac{(p+q)}{p^{3}[s-1]_{p, q}[s-2]_{p, q}} \sum_{\ell=0}^{\infty} \mathcal{Q}_{s, p, q}(u) .
\end{aligned}
$$

Now by separating the even and odd terms and applying $\theta_{\ell}$ from (1.5), i.e., taking $\ell=2 m$ and $\ell=2 m+1$ for all $m=0,1,2, \ldots$, we have

$$
\begin{aligned}
\mathcal{P}_{s, p, q}^{\tau}\left(t^{2} ; u\right) \geq & \frac{q^{3}[s]_{p, q}^{2}}{[s-1]_{p, q}[s-2]_{p, q}} \sum_{\ell=0}^{\infty} \mathcal{Q}_{s, p, q}(u)\left(\frac{p^{\ell+2 \tau \theta_{\ell}}-q^{\ell+2 \tau \theta_{\ell}}}{p^{\ell-1}\left(p^{s}-q^{s}\right)}\right)^{2} \\
& +\frac{q\left(q+[2]_{p, q}\right)[s]_{p, q}}{[s-1]_{p, q}[s-2]_{p, q}} \sum_{\ell=0}^{\infty} \mathcal{Q}_{s, p, q}(u)\left(\frac{p^{\ell+2 \tau \theta_{\ell}}-q^{\ell+2 \tau \theta_{\ell}}}{p^{\ell-1}\left(p^{s}-q^{s}\right)}\right) \\
& +\frac{[2]_{p, q}}{[s-1]_{p, q}[s-2]_{p, q}} \\
= & \frac{q^{3}[s]_{p, q}^{2}}{[s-1]_{p, q}[s-2]_{p, q}} D_{s, p, q}\left(t^{2} ; u\right)+\frac{q\left(q+[2]_{p, q}\right)[s]_{p, q}}{[s-1]_{p, q}[s-2]_{p, q}} D_{s, p, q}(t ; u) \\
& +\frac{[2]_{p, q}}{[s-1]_{p, q}[s-2]_{p, q}} .
\end{aligned}
$$

Similarly,

$$
\begin{aligned}
\mathcal{P}_{s, p, q}^{\tau}\left(t^{2} ; u\right) \leq & \frac{[s]_{p, q}^{2}}{[s-1]_{p, q}[s-2]_{p, q}} D_{s, p, q}\left(t^{2} ; u\right)+\frac{\left(1+[2]_{p, q}\right)[s]_{p, q}}{[s-1]_{p, q}[s-2]_{p, q}} D_{s, p, q}(t ; u) \\
& +\frac{[2]_{p, q}}{[s-1]_{p, q}[s-2]_{p, q}} .
\end{aligned}
$$

This completes the proof of Lemma 2.2.

Lemma 2.3 Let $\Phi_{j}=(t-u)^{j}$ for $j=1,2$, then we have following inequalities:

1. $\mathcal{P}_{s, p, q}^{\tau}\left(\Phi_{1} ; u\right) \leq\left(\frac{[s]_{p, q}}{[s-1]_{p, q}}-1\right) u+\frac{1}{[s-1]_{p, q}}, \quad$ for $s>1, s \in \mathbb{N}$,

2. $\mathcal{P}_{s, p, q}^{\tau}\left(\Phi_{2} ; u\right) \leq\left(\frac{[s]_{p, q}^{2}}{[s-1]_{p, q}[s-2]_{p, q}}-\frac{2[s]_{p, q}}{[s-1]_{p, q}}+1\right) u^{2}$

$$
\begin{aligned}
& +\frac{1}{[s-1]_{p, q}}\left(\frac{[s]_{p, q}}{[s-2]_{p, q}}\left(1+[2]_{p, q}+[1+2 \tau]_{p, q}\right)-2\right) u \\
& +\frac{[2]_{p, q}}{[s-1]_{p, q}[s-2]_{p, q}}, \quad \text { for } s>2, s \in \mathbb{N} .
\end{aligned}
$$

\section{Approximation results}

Let us denote by $C_{B}[0, \infty)$ the set of all bounded and continuous functions defined on $[0, \infty)$, equipped with the norm $\|f\|_{C_{B}}=\sup _{u \geq 0}|f(u)|$. We write

$$
\mathcal{L}:=\left\{f: \lim _{u \rightarrow \infty} \frac{f(u)}{1+u^{2}} \text { exits }\right\}
$$




$$
B_{\sigma}[0, \infty):=\left\{f:|f(u)| \leq \mathcal{M}_{f} \sigma(u)\right\}
$$

where $\mathcal{M}_{f}$ is a constant depending on $f$, and $\sigma$ is the weight function with $\sigma(u)=1+u^{2}$. Moreover,

$$
\begin{aligned}
& C_{\sigma}[0, \infty):=B_{\sigma}[0, \infty) \cap C[0, \infty), \\
& C_{\sigma}^{k}[0, \infty):=\left\{f: f \in C_{\sigma}[0, \infty) \text { and } \lim _{u \rightarrow \infty} \frac{f(u)}{\sigma(u)}=k<\infty\right\} .
\end{aligned}
$$

Note that $C_{\sigma}[0, \infty)$ is a normed space with the norm given by $\|f\|_{\sigma}=\sup _{u \geq 0} \frac{|f(u)|}{\sigma(u)}$.

Theorem 3.1 Take the sequences of positive numbers $q=q_{s}, p=p_{s}$ satisfying $q_{s} \in(0,1)$, $p_{s} \in\left(q_{s}, 1\right]$ such that $\lim _{s \rightarrow \infty} q_{s}=1, \lim _{s \rightarrow \infty} p_{s}=1$. Then, $\mathcal{P}_{s, p_{s}, q_{s}}^{\tau}$ is uniformly convergent on each compact subset of $[0, \infty)$ and such that

$$
\lim _{s \rightarrow \infty} \mathcal{P}_{s, p_{s}, q_{s}}^{\tau}(f ; u)=f(u)
$$

where $f \in C[0, \infty) \cap \mathcal{L}$.

Proof To prove the uniform convergence on each compact subset of $[0, \infty)$, it is obvious from the well-known Korovkin's theorem [23] that $\lim _{s \rightarrow \infty} \mathcal{P}_{s, p_{s}, q_{s}}^{\ell, \tau}\left(t^{\eta} ; u\right)=u^{\eta}$ for $\eta=0,1,2$. Whenever, $q_{s}=1, p_{s}=1$ as $s \rightarrow \infty$, then clearly for all $i=1,2$ we have $\frac{1}{[s-i]_{s, q} q_{s}} \rightarrow 0$, $\frac{[s]_{p_{s}, q_{s}}}{[s-i]_{p_{s}, q_{s}}} \rightarrow 1$, which imply that

$$
\lim _{s \rightarrow \infty} \mathcal{P}_{s, p_{s}, q_{s}}^{\tau}(1 ; u)=1, \quad \lim _{s \rightarrow \infty} \mathcal{P}_{s, p_{s}, q_{s}}^{\tau}(t ; u)=u, \quad \lim _{s \rightarrow \infty} \mathcal{P}_{s, p_{s}, q_{s}}^{\tau}\left(t^{2} ; u\right)=u^{2} .
$$

Theorem 3.2 For each $f \in C_{\sigma}^{k}[0, \infty)$, consider the sequences of positive numbers $0<q_{s}<$ $p_{s} \leq 1$ such that $\lim _{s \rightarrow \infty} q_{s}=1, \lim _{s \rightarrow \infty} p_{s}=1$. Then the operators $\mathcal{P}_{s, p_{s}, q_{s}}^{\tau}$ satisfy

$$
\lim _{s \rightarrow \infty}\left\|\mathcal{P}_{s, p_{s}, q_{s}}^{\tau}(f)-f\right\|_{\sigma}=0
$$

Proof We take $f(t)=t^{\eta}$ with $\eta=0,1,2$. From Theorem 3.1 , since $\mathcal{P}_{s, p_{s}, q_{s}}^{\tau}\left(t^{\eta} ; u\right)$ is uniformly convergent to $u^{\eta}$ for all $\eta=0,1,2$, and applying Lemma 2.2, we conclude that

$$
\lim _{s \rightarrow \infty}\left\|\mathcal{P}_{s, p_{s}, q_{s}}^{\tau}(1)-1\right\|_{\sigma}=0
$$

For $\eta=1$,

$$
\begin{aligned}
\left\|\mathcal{P}_{s, p_{s}, q_{s}}^{\tau}(t)-u\right\|_{\sigma} & =\sup _{u \geq 0} \frac{\left|\mathcal{P}_{s, p_{s}, q_{s}}^{\tau}(t ; u)-u\right|}{1+u^{2}} \\
& \leq\left(\frac{[s]_{p_{s}, q_{s}}}{[s-1]_{p_{s}, q_{s}}}-1\right) \sup _{u \geq 0} \frac{u}{1+u}+\frac{1}{[s-1]_{p_{s}, q_{s}}} \sup \frac{1}{u \geq 0} \frac{1+u}{}
\end{aligned}
$$

Then

$$
\lim _{s \rightarrow \infty}\left\|\mathcal{P}_{s, p_{s}, q_{s}}^{\tau}(t)-u\right\|_{\sigma}=0
$$


Similarly, if we take $\eta=2$, then

$$
\begin{aligned}
\left\|\mathcal{P}_{s, p_{s}, q_{s}}^{\tau}\left(t^{2}\right)-u^{2}\right\|_{\sigma}= & \sup _{u \geq 0} \frac{\left|\mathcal{P}_{s, p_{s}, q_{s}}^{\tau}\left(t^{2} ; u\right)-u^{2}\right|}{1+u^{2}} \\
\leq & \left(\frac{[s]_{p_{s}, q_{s}}^{2}}{[s-1]_{p_{s}, q_{s}}[s-2]_{p, q}}-1\right) \sup _{u \geq 0} \frac{u^{2}}{1+u^{2}} \\
& +\frac{[s]_{p_{s}, q_{s}}}{[s-1]_{p_{s}, q_{s}}[s-2]_{p_{s}, q_{s}}}\left(1+[2]_{p_{s}, q_{s}}+[1+2 \tau]_{p_{s}, q_{s}}\right) \sup _{u \geq 0} \frac{u}{1+u^{2}} \\
& +\frac{[2]_{p_{s}, q_{s}}}{[s-1]_{p_{s}, q_{s}}[s-2]_{p, q}} \sup \frac{1}{1+u^{2}}, \\
\lim _{s \rightarrow \infty}\left\|\mathcal{P}_{s, p_{s}, q_{s}}^{\tau}\left(t^{2}\right)-u^{2}\right\|_{\sigma} & =0 .
\end{aligned}
$$

This completes the proof.

Let

$$
\omega_{\mu}(f ; \delta)=\sup _{|t-u| \leq \delta} \sup _{u, t \in[0, \mu]}|f(t)-f(u)| .
$$

It is obvious that $\lim _{\delta \rightarrow 0+} \omega_{\mu}(f ; \delta)=0$ and for $f \in C[0, \infty)$,

$$
|f(t)-f(u)| \leq\left(\frac{|t-u|}{\delta}+1\right) \omega_{\mu}(f ; \delta)
$$

Theorem 3.3 Let $f \in C_{\sigma}[0, \infty)$, and $0<q_{s}<p_{s} \leq 1$ be such that $\lim _{s \rightarrow \infty} q_{s}=1, \lim _{s \rightarrow \infty} p_{s}=$ 1. Moreover, suppose $\omega_{\mu}(f ; \delta)$ is defined by (3.5) on the interval $[0, \mu+1] \subset[0, \infty)$, for $\mu>0$. Then for every $s>2$, we get

$$
\left|\mathcal{P}_{s, p_{s}, q_{s}}^{\tau}(f ; u)-f(u)\right| \leq 2 \omega_{\mu+1}\left(f ; \delta_{s}(u)\right)+6 \mathcal{C}_{f}\left(1+\mu^{2}\right)\left(\delta_{s}(u)\right)^{2}
$$

where $\mathcal{C}_{f}$ is a constant depending only on $f$ and $\delta_{s}(u)=\sqrt{\mathcal{P}_{s, p_{s}, q_{s}}^{\tau}\left(\Phi_{2} ; u\right)}$.

Proof For $u \in[0, \mu]$ and $t \leq \mu+1$, with $\mu>0$, we have

$$
|f(t)-f(u)| \leq \mathcal{C}_{f}\left(2+u^{2}+t^{2}\right) \leq 6 \mathcal{C}_{f}\left(1+\mu^{2}\right)(t-u)^{2} .
$$

Furthermore, for any $\delta>0, u \in[0, \mu]$, and $t>\mu+1$, with $\mu>0$,

$$
|f(t)-f(u)| \leq \omega_{\mu+1}(f ;|t-u|) \leq\left(1+\frac{|t-u|}{\delta}\right) \omega_{\mu+1}(f ; \delta) .
$$

From (3.7) and (3.8), we have

$$
|f(t)-f(u)| \leq 6 \mathcal{C}_{f}\left(1+\mu^{2}\right)(t-u)^{2}+\left(1+\frac{|t-u|}{\delta}\right) \omega_{\mu+1}(f ; \delta) .
$$


Applying operators $\mathcal{P}_{s, p_{s}, q_{s}}^{\tau}$ and the well-known Cauchy-Schwartz inequality, we have

$$
\begin{aligned}
\mathcal{P}_{s, p_{s}, q_{s}}^{\tau}(|f(t)-f(u)| ; u) \leq & 6 \mathcal{C}_{f}\left(1+\mu^{2}\right) \mathcal{P}_{s, p_{s}, q_{s}}^{\tau}\left(\Phi_{2} ; u\right) \\
& +\mathcal{P}_{s, p_{s}, q_{s}}^{\tau}\left(1+\frac{|t-u|}{\delta} ; u\right) \omega_{\mu+1}(f ; \delta) .
\end{aligned}
$$

Moreover, for any $g \in C_{\sigma}[0, \infty)$, we know

$$
\begin{aligned}
\mathcal{P}_{s, p_{s}, q_{s}}^{\tau}(g ; u)-g(u) & =\mathcal{P}_{s, p_{s}, q_{s}}^{\tau}(g ; u)-g(u) \mathcal{P}_{s, p_{s}, q_{s}}^{\tau}(1 ; u) \\
& =\mathcal{P}_{s, p_{s}, q_{s}}^{\tau}(g(t)-g(u) ; u) \\
& \leq \mathcal{P}_{s, p_{s}, q_{s}}^{\tau}(|g(t)-g(u)| ; u) .
\end{aligned}
$$

Therefore,

$$
\begin{aligned}
\left|\mathcal{P}_{s, p_{s}, q_{s}}^{\tau}(f ; u)-f(u)\right| \leq & 6 \mathcal{C}_{f}\left(1+\mu^{2}\right) \mathcal{P}_{s, p_{s}, q_{s}}^{\tau}(t-u)^{2} \\
& +\left(1+\frac{1}{\delta} \mathcal{P}_{s, p_{s}, q_{s}}^{\tau}(|t-u| ; u)\right) \omega_{\mu+1}(f ; \delta) \\
\leq & 6 \mathcal{C}_{f}\left(1+\mu^{2}\right) \mathcal{P}_{s, p_{s}, q_{s}}^{\tau}\left(\Phi_{2} ; u\right) \\
& +\left(1+\frac{1}{\delta} \mathcal{P}_{s, p_{s}, q_{s}}^{\tau}\left(\Phi_{2} ; u\right)^{\frac{1}{2}}\right) \omega_{\mu+1}(f ; \delta)
\end{aligned}
$$

where

$$
\mathcal{P}_{s, p_{s}, q_{s}}^{\tau}(|t-u| ; u) \leq \mathcal{P}_{s, p_{s}, q_{s}}^{\tau}(1 ; u)^{\frac{1}{2}} \mathcal{P}_{s, p_{s}, q_{s}}^{\tau}\left((t-u)^{2} ; u\right)^{\frac{1}{2}}=\mathcal{P}_{s, p_{s}, q_{s}}^{\tau}\left(\Phi_{2} ; u\right)^{\frac{1}{2}}
$$

Finally, if we choose $\delta=\delta_{s}(u)=\sqrt{\mathcal{P}_{s, p_{s}, q_{s}}^{\tau}\left(\Phi_{2} ; u\right)}$, then we get the desired result.

\section{Rate of convergence}

In 1963, to measure the smoothness, a mathematical formula of a certain functional was given by Peetre [41]. For all $\delta>0$ and $f \in C[0, \infty)$, Peetre defined the $K$-functional, which we write as $K_{2}(f ; \delta)$. The formulas below give its definition, as well as a bound for some constant $\mathcal{C}>0$ and the second-order modulus of continuity $\omega_{2}(f ; \delta)$ defined as follows:

$$
\begin{aligned}
& K_{2}(f ; \delta)=\inf _{u \geq 0}\left\{\left(\|f-\psi\|_{C_{B}[0, \infty)}+\delta\left\|\psi^{\prime \prime}\right\|_{C_{B}[0, \infty)}\right): \psi \in C_{B}^{2}[0, \infty)\right\}, \\
& K_{2}(f ; \delta) \leq \mathcal{C}\left\{\omega_{2}(f ; \sqrt{\delta})+\min (1, \delta)\|f\|_{C_{B}[0, \infty)}\right\} \\
& \omega_{2}(f ; \delta)=\sup _{0<v<\delta} \sup _{u \geq 0}|f(u+2 v)-2 f(u+v)+f(u)| .
\end{aligned}
$$

Theorem 4.1 Let $q=q_{s}, p=p_{s}$ with $q_{s} \in(0,1), p_{s} \in\left(q_{s}, 1\right]$ and $\mathcal{R}_{s, p, q}^{\tau}(f ; u)=\mathcal{P}_{s, p, q}^{\tau}(f ; u)+$ $f(u)-f\left(\frac{[s]_{p, q} u+1}{[s-1]_{p, q}}\right)$. Then, for every $\psi \in C_{B}^{2}[0, \infty)$ and $s>2$, we have

$$
\left|\mathcal{R}_{s, p_{s}, q_{s}}^{\tau}(\psi ; u)-\psi(u)\right| \leq \chi_{n}(u)\left\|\psi^{\prime \prime}\right\|
$$

where $\chi_{n}(u)=\delta_{s}^{2}(u)+\left(\mathcal{P}_{s, p, q}\left(\Phi_{1} ; u\right)\right)^{2}$, in which $\delta_{s}(u)$ is defined in Theorem 3.3 and $\mathcal{P}_{s, p, q}\left(\Phi_{1} ; u\right)$ is defined by Lemma 2.3 . 
Nasiruzzaman et al. Journal of Inequalities and Applications

(2021) 2021:6

Page 9 of 13

Proof Let $\psi \in C_{B}^{2}[0, \infty)$. We easily get $\mathcal{R}_{s, p_{s}, q_{s}}^{\tau}(1 ; u)=1$ and

$$
\mathcal{R}_{s, p_{s}, q_{s}}^{\tau}(t ; u)=\mathcal{P}_{s, p_{s}, q_{s}}^{\tau}(t ; u)+u-\left(\frac{[s]_{p_{s}, q_{s}} u+1}{[s-1]_{p_{s}, q_{s}}}\right)=u
$$

Also

$$
\begin{aligned}
& \left\|\mathcal{P}_{s, p_{s}, q_{s}}^{\tau}(f ; u)\right\| \leq\|f\|, \\
& \left|\mathcal{R}_{s, p_{s}, q_{s}}^{\tau}(f ; u)\right| \leq\left|\mathcal{P}_{s, p_{s}, q_{s}}^{\tau}(f ; u)\right|+|f(u)|-\left|f\left(\frac{[s]_{p_{s}, q_{s}} u+1}{[s-1]_{p_{s}, q_{s}}}\right)\right| \leq 3\|f\| .
\end{aligned}
$$

From the Taylor series expansion, we have

$$
\psi(t)=\psi(u)+(t-u) \psi^{\prime}(u)+\int_{u}^{t}(t-\alpha) \psi^{\prime \prime}(\alpha) \mathrm{d} \alpha .
$$

Applying the operator $\mathcal{R}_{s, p_{s}, q_{s}}^{\tau}$, we conclude that

$$
\begin{aligned}
\mathcal{R}_{s, p_{s}, q_{s}}^{\tau}(\psi ; u)-\psi(u)= & \psi^{\prime}(u) \mathcal{R}_{s, p_{s}, q_{s}}^{\tau}(t-u ; u)+\mathcal{R}_{s, p_{s}, q_{s}}^{\tau}\left(\int_{u}^{t}(t-\alpha) \psi^{\prime \prime}(\alpha) \mathrm{d} \alpha ; u\right) \\
= & \mathcal{R}_{s, p_{s}, q_{s}}^{\tau}\left(\int_{u}^{t}(t-\alpha) \psi^{\prime \prime}(\alpha) \mathrm{d} \alpha ; u\right) \\
= & \mathcal{P}_{s, p_{s}, q_{s}}^{\tau}\left(\int_{u}^{t}(t-\alpha) \psi^{\prime \prime}(\alpha) \mathrm{d} \alpha ; u\right) \\
& -\int_{u}^{\frac{[s]_{s}, q_{s} u+1}{[s-1] p_{s}, q_{s}}}\left(\frac{[s]_{p_{s}, q_{s}} u+1}{[s-1]_{p_{s}, q_{s}}}-\alpha\right) \psi^{\prime \prime}(\alpha) \mathrm{d} \alpha \\
\left|\mathcal{R}_{s, p_{s}, q_{s}}^{\tau}(\psi ; u)-\psi(u)\right| \leq & \left|\mathcal{P}_{s, p_{s}, q_{s}}^{\tau}\left(\int_{u}^{t}(t-\alpha) \psi^{\prime \prime}(\alpha) \mathrm{d} \alpha ; u\right)\right| \\
& +\left|\int_{u}^{\frac{[s] p_{s}, q_{s} u+1}{\left[s-1 p_{s}, q_{s}\right.}}\left(\frac{[s]_{p_{s}, q_{s}} u+1}{[s-1]_{p_{s}, q_{s}}}-\alpha\right) \psi^{\prime \prime}(\alpha) \mathrm{d} \alpha\right| .
\end{aligned}
$$

Since

$$
\left|\int_{u}^{t}(t-\alpha) \psi^{\prime \prime}(\alpha) \mathrm{d} \alpha\right| \leq(t-u)^{2}\left\|\psi^{\prime \prime}\right\|
$$

we conclude that

$$
\left|\int_{u}^{\frac{[s]_{p_{s}, q_{s} u+1}}{[s-1]_{p_{s}, q_{s}}}}\left(\frac{[s]_{p_{s}, q_{s}} u+1}{[s-1]_{p_{s}, q_{s}}}-\alpha\right) \psi^{\prime \prime}(\alpha) \mathrm{d} \alpha\right| \leq\left(\frac{[s]_{p_{s}, q_{s}} u+1}{[s-1]_{p_{s}, q_{s}}}-u\right)^{2}\left\|\psi^{\prime \prime}\right\| .
$$

Hence,

$$
\left|\mathcal{R}_{s, p_{s}, q_{s}}^{\tau}(\psi ; u)-\psi(u)\right| \leq\left\{\mathcal{P}_{s, p_{s}, q_{s}}^{\tau}\left((t-u)^{2} ; u\right)+\left(\frac{[s]_{p_{s}, q_{s}} u+1}{[s-1]_{p_{s}, q_{s}}}-u\right)^{2}\right\}\left\|\psi^{\prime \prime}\right\| .
$$

Thus we complete the proof. 
Theorem 4.2 Let $q=q_{s}, p=p_{s}$ with $q_{s} \in(0,1), p_{s} \in\left(q_{s}, 1\right]$ and $f \in C_{B}[0, \infty)$. Then, for every $\psi \in C_{B}^{2}[0, \infty)$ and $s>2$ there exits a positive constant $\mathcal{C}>$ satisfying the inequality

$$
\begin{aligned}
\left|\mathcal{P}_{s, p_{s}, q_{s}}^{\tau}(f ; u)-f(u)\right| \leq & \mathcal{A}\left\{\omega_{2}\left(f ; \frac{\sqrt{\chi_{s}(u)}}{2}\right)+\min \left(1, \frac{\chi_{s}(u)}{4}\right)\|f\|\right\} \\
& +\omega\left(f ;\left|\mathcal{P}_{s, p_{s}, q_{s}}\left(\Phi_{1} ; u\right)\right|\right) .
\end{aligned}
$$

Proof For all $f \in C_{B}[0, \infty)$ and $\psi \in C_{B}^{2}[0, \infty)$, it is very easy to see the result from Theorem 4.1. Indeed,

$$
\begin{aligned}
\left|\mathcal{R}_{s, p_{s}, q_{s}}^{\tau}(f ; u)-f(u)\right|= & \left|\mathcal{R}_{s, p_{s}, q_{s}}^{\tau}(f ; u)-f(u)+f\left(\frac{[s]_{p_{s}, q_{s}} u+1}{[s-1]_{p, q}}\right)-f(u)\right| \\
\leq & \left|\mathcal{R}_{s, p_{s}, q_{s}}^{\tau}(f-\psi ; u)\right|+\left|\mathcal{R}_{s, p_{s}, q_{s}}^{\tau}(\psi ; u)-\psi(u)\right| \\
& +|\psi(u)-f(u)|+\left|f\left(\frac{[s]_{p_{s}, q_{s}} u+1}{[s-1]_{p_{s}, q_{s}}}\right)-f(u)\right| \\
\leq & 4\|f-\psi\|+\chi_{s}(u)\left\|\psi^{\prime \prime}\right\| \\
& +\omega\left(f ;\left|\left(\frac{[s]_{p_{s}, q_{s}}}{[s-1]_{p_{s}, q_{s}}}-1\right) u+\frac{1}{[s-1]_{p_{s}, q_{s}}}\right|\right) .
\end{aligned}
$$

By taking the infimum over all $\psi \in C_{B}^{2}[0, \infty)$ and using (4.1), we get

$$
\begin{aligned}
\left|\mathcal{R}_{s, p_{s}, q_{s}}^{\tau}(f ; u)-f(u)\right| \leq & 4 K_{2}\left(f ; \frac{\chi_{s}(u)}{4}\right)+\omega\left(f ;\left|\left(\frac{[s]_{p_{s}, q_{s}}}{[s-1]_{p_{s}, q_{s}}}-1\right) u+\frac{1}{[s-1]_{p_{s}, q_{s}}}\right|\right) \\
\leq & \mathcal{A}\left\{\omega_{2}\left(f ; \frac{\sqrt{\chi_{s}(u)}}{2}\right)+\min \left(1 ; \frac{\chi_{s}(u)}{4}\right)\|f\|\right\} \\
& +\omega\left(f ;\left|\left(\frac{[s]_{p_{s}, q_{s}}}{[s-1]_{p_{s}, q_{s}}}-1\right) u+\frac{1}{[s-1]_{p_{s}, q_{s}}}\right|\right) .
\end{aligned}
$$

We consider the following Lipschitz-type maximal function [24] and obtain the local approximation. For $f \in C[0, \infty], 0<\kappa \leq 1$ and $t, u \geq 0$, we recall that

$$
\operatorname{Lip}_{M}(\kappa)=\left\{f:|f(t)-f(u)| \leq M|t-u|^{\kappa}\right\} .
$$

Theorem 4.3 For all $\kappa \in(0,1], s>2$, and $f \in C_{B}[0, \infty)$, we have

$$
\left|\mathcal{P}_{s, p_{s}, q_{s}}^{\tau}(f ; u)-f(u)\right| \leq M\left(\delta_{s}(u)\right)^{\kappa},
$$

where $\delta_{s}(u)$ is given in Theorem 3.3 .

Proof We prove the claim by applying (4.4) and the well-known Hölder's inequality:

$$
\begin{aligned}
\left|\mathcal{P}_{s, p_{s}, q_{s}}^{\tau}(f ; u)-f(u)\right| & \leq \mathcal{P}_{s, p_{s}, q_{s}}^{\tau}(|f(t)-f(u)| ; u) \\
& \leq M \mid \mathcal{P}_{s, p_{s}, q_{s}}^{\tau}\left(|t-u|^{\kappa} ; u\right)
\end{aligned}
$$




$$
\begin{aligned}
& \leq M\left(\mathcal{P}_{s, p_{s}, q_{s}}^{\tau}(1 ; u)\right)^{\frac{2-\kappa}{2}}\left(\mathcal{P}_{s, p_{s}, q_{s}}^{\tau}\left(|t-u|^{2} ; u\right)\right)^{\frac{\kappa}{2}} \\
& =M\left(\mathcal{P}_{s, p_{s}, q_{s}}^{\tau}\left(\Phi_{2} ; u\right)\right)^{\frac{\kappa}{2}} .
\end{aligned}
$$

This gives the desired result.

We denote

$$
\begin{aligned}
& C_{B}^{2}[0, \infty)=\left\{\psi: \psi \in C_{B}[0, \infty) \text { and } \psi^{\prime}, \psi^{\prime \prime} \in C_{B}[0, \infty)\right\}, \\
& \|\psi\|_{C_{B}^{2}[0, \infty)}=\|\psi\|_{C_{B}[0, \infty)}+\left\|\psi^{\prime}\right\|_{C_{B}[0, \infty)}+\left\|\psi^{\prime \prime}\right\|_{C_{B}[0, \infty)}, \\
& \|\psi\|_{C_{B}[0, \infty)}=\sup _{u \geq 0}|\psi(u)| .
\end{aligned}
$$

Theorem 4.4 Let the positive sequences of numbers $0<q_{s}<p_{s} \leq 1$ satisfy $\lim _{s \rightarrow \infty} q_{s}=1$, $\lim _{s \rightarrow \infty} p_{s}=1$. Then for all $\psi \in C_{B}^{2}[0, \infty)$, the operators $\mathcal{P}_{s, p_{s}, q_{s}}^{\tau}$ have the property

$$
\left|\mathcal{P}_{s, p_{s}, q_{s}}^{\tau}(\psi ; u)-\psi(u)\right| \leq \Theta_{s}(u)\|\psi\|_{C_{B}^{2}[0, \infty)},
$$

where $\Theta_{s}(u)=\sqrt{\delta_{s}(u)}+\frac{\left(\delta_{s}(u)\right)^{2}}{2}$.

Proof Let $\psi \in C_{B}^{2}[0, \infty)$. Then

$$
\psi(t)=\psi(u)+\psi^{\prime}(u)(t-u)+\psi^{\prime \prime}(\varphi) \frac{(t-u)^{2}}{2} \text { for } \varphi \in(u, t),
$$

where if we take

$$
\begin{aligned}
& \mathcal{S}=\sup _{u \geq 0}\left|\psi^{\prime}(u)\right|=\left\|\psi^{\prime}\right\|_{C_{B}[0, \infty)} \leq\|\psi\|_{C_{B}^{2}[0, \infty)}, \\
& \mathcal{T}=\sup _{u \geq 0}\left|\psi^{\prime \prime}(u)\right|=\left\|\psi^{\prime \prime}\right\|_{C_{B}[0, \infty)} \leq\|\psi\|_{C_{B}^{2}[0, \infty)},
\end{aligned}
$$

then we have

$$
\begin{aligned}
|\psi(t)-\psi(u)| & \leq \mathcal{S}|t-u|+\frac{1}{2} \mathcal{T}(t-u)^{2} \\
& \leq\left(|t-u|+\frac{1}{2}(t-u)^{2}\right)\|\psi\|_{C_{B}^{2}[0, \infty)} .
\end{aligned}
$$

Therefore

$$
\begin{aligned}
\left|\mathcal{P}_{s, p_{s}, q_{s}}^{\tau}(\psi ; u)-\psi(u)\right| & \leq\left(\mathcal{P}_{s, p_{s}, q_{s}}^{\tau}(|t-u| ; u)+\frac{1}{2} \mathcal{P}_{s, p_{s}, q_{s}}^{\tau}\left((t-u)^{2} ; u\right)\right)\|\psi\|_{C_{B}^{2}[0, \infty)} \\
& \leq\left(\left(\mathcal{P}_{s, p_{s}, q_{s}}^{\tau}\left(\Phi_{2} ; u\right)\right)^{\frac{1}{2}}+\frac{1}{2} \mathcal{P}_{s, p_{s}, q_{s}}^{\tau}\left(\Phi_{2} ; u\right)\right)\|\psi\|_{C_{B}^{2}[0, \infty)} .
\end{aligned}
$$

This completes the proof of Theorem 4.4. 


\title{
5 Conclusion
}

We constructed a $(p, q)$-variant of Szász operators by using the Beta functions of the second kind by introducing the Dunkl generalization. We obtained the approximation results involving local and global approximations in Korovkin's and weighted Korovkin's spaces. We applied some techniques of earlier investigation and discussed the convergence of operators by employing the modulus of continuity, Lipschitz class and Peetre's $K$-functionals.

\author{
Acknowledgements \\ NA \\ Funding \\ NA \\ Availability of data and materials \\ NA \\ Competing interests \\ The authors declare that they have no competing interests.
}

\section{Authors' contributions}

The authors contributed equally and significantly in writing this paper. All authors read and approved the final manuscript.

\section{Author details}

${ }^{1}$ Department of Mathematics, Faculty of Science, University of Tabuk, PO Box 4279, Tabuk 71491, Saudi Arabia. ${ }^{2}$ Operator Theory and Applications Research Group, Department of Mathematics, Faculty of Science, King Abdulaziz University, Jeddah, Saudi Arabia. ${ }^{3}$ Department of Medical Research, China Medical University Hospital, China Medical University (Taiwan), Taichung, Taiwan. ${ }^{4}$ Department of Mathematics, Aligarh Muslim University, Aligarh 202002, India.

\section{Publisher's Note}

Springer Nature remains neutral with regard to jurisdictional claims in published maps and institutional affiliations.

Received: 14 May 2020 Accepted: 10 December 2020 Published online: 04 January 2021

\section{References}

1. Acar, T.: (p,q)-Generalization of Szász-Mirakyan operators. Math. Methods Appl. Sci. 39(10), 2685-2695 (2016)

2. Acar, T., Agrawal, P.N., Kumar, A.S.: On a modification of $(p, q)$-Szász-Mirakyan operators. Complex Anal. Oper. Theory 12(1), 155-167 (2018)

3. Acar, T., Aral, A., Mohiuddine, S.A.: On Kantorovich modification of (p, q)-Baskakov operators. J. Inequal. Appl. 2016, 98 (2016)

4. Acar, T., Aral, A., Mohiuddine, S.A.: On Kantorovich modification of (p, q)-Bernstein operators. Iran. J. Sci. Technol., Trans. A, Sci. 42, 1459-1464 (2018)

5. Acar, T., Aral, A., Mohiuddine, S.A.: Approximation by bivariate $(p, q)$-Bernstein-Kantorovich operators. Iran. J. Sci. Technol. Trans. A, Sci. 42(2), 655-662 (2018)

6. Acar, T., Aral, A., Mursaleen, M.: Approximation by Baskakov-Durrmeyer operators based on $(p, q)$-integers. Math. Slovaca 68(4), 897-906 (2018)

7. Acar, T., Aral, A., Raşa, l.: Positive linear operators preserving $\tau$ and $\tau^{2}$. Constr. Math. Anal. 2(3), 98-102 (2019)

8. Acar, T., Mohiuddine, S.A., Mursaleen, M.: Approximation by $(p, q)$-Baskakov-Durrmeyer-Stancu operators. Complex Anal. Oper. Theory 12(6), 1453-1468 (2018)

9. Acar, T., Mursaleen, M., Mohiuddine, S.A.: Stancu type (p,q)-Szász-Mirakyan-Baskakov operators. Commun. Fac. Sci. Univ. Ank. Sér. A1 Math. Stat. 67(1), 116-128 (2018)

10. Alotaibi, A., Nasiruzzaman, M., Mursaleen, M.: A Dunkl type generalization of Szász operators via post-quantum calculus. J. Inequal. Appl. 2018, 287 (2018)

11. Bin Jebreen, H., Mursaleen, M., Naaz, A.: Approximation by quaternion ( $p, q)$-Bernstein polynomials and Voronovskaja type result on compact disk. Adv. Differ. Equ. 2018, 448 (2018)

12. De Sole, A., Kac, V.G.: On integral representations of $q$-gamma and $q$-beta functions. Atti Accad. Naz. Lincei, Rend. Lincei, Mat. Appl. 16, 11-29 (2005)

13. İçöz, G., Çekim, B.: Dunkl generalization of Szász operators via q-calculus. J. Inequal. Appl. 2015, 284 (2015)

14. Ince Illarslan, H.G., Acar, T.: Approximation by bivariate $(p, q)$-Baskakov-Kantorovich operators. Georgian Math. J. 25(3), 397-407 (2018)

15. Kadak, $\mathrm{U}$ : On weighted statistical convergence based on $(p, q)$-integers and related approximation theorems for functions of two variables. J. Math. Anal. Appl. 443(2), 752-764 (2016)

16. Kadak, U.: Weighted statistical convergence based on generalized difference operator involving $(p, q)$-gamma function and its applications to approximation theorems. J. Math. Anal. Appl. 448(2), 1633-1650 (2017)

17. Kadak, U., Mishra, V.N., Pandey, S.: Chlodowsky type generalization of $(p, q)$-Szász operators involving Brenke type polynomials. Rev. R. Acad. Cienc. Exactas Fís. Nat., Ser. A Mat. 112(4), 1443-1462 (2018) 
18. Kadak, U., Mohiuddine, S.A.: Generalized statistically almost convergence based on the difference operator which includes the $(p, q)$-Gamma function and related approximation theorems. Results Math. 73(9) (2018). https://doi.org/10.1007/s00025-018-0789-6

19. Khan, A., Sharma, V.: Statistical approximation by $(p, q)$-analogue of Bernstein-Stancu operators. Azerb. J. Math. 8(2), 100-121 (2018)

20. Khan, K., Lobiyal, D.K.: Bézier curves based on Lupaş $(p, q)$-analogue of Bernstein functions in CAGD. J. Comput. Appl. Math. 317, 458-477 (2017)

21. Khan, K., Lobiyal, D.K., Kilicman, A.: Bézier curves and surfaces based on modified Bernstein polynomials. Azerb. J. Math. 9(1), 3-21 (2019)

22. Kilicman, A., Ayman Mursaleen, M., Al-Abied, A.H.H.: Stancu type Baskakov-Durrmeyer operators and approximation properties. Mathematics 8, Article ID 1164 (2020). https://doi.org/10.3390/math8071164

23. Korovkin, P.P.: On convergence of linear operators in the space of continuous functions. Dokl. Akad. Nauk SSSR 90 961-964 (1953) (Russian)

24. Lenze, B.: On Lipschitz type maximal functions and their smoothness spaces. Nederl. Akad. Indag. Math. 50, 53-63 (1988)

25. Lupaş, A.: A q-analogue of the Bernstein operator. Univ. Cluj-Napoca Seminar Numer. Stat., Calculus 9, 85-92 (1987)

26. Maurya, R., Sharma, H., Gupta, C.: Approximation properties of Kantorovich type modifications of (p,q)-Meyer-König-Zeller operators. Constr. Math. Anal. 1(1), 58-72 (2018)

27. Milovanovic, G.V., Mursaleen, M., Nasiruzzaman, M.: Modified Stancu type Dunkl generalization of Szász-Kantorovich operators. Rev. R. Acad. Cienc. Exactas Fís. Nat., Ser. A Mat. 112, 135-151 (2018)

28. Mohiuddine, S.A., Acar, T., Alghamdi, M.A.: Genuine modified Bernstein-Durrmeyer operators. J. Inequal. Appl. 2018, $104(2018)$

29. Mohiuddine, S.A., Acar, T., Alotaibi, A.: Construction of a new family of Bernstein-Kantorovich operators. Math. Methods Appl. Sci. 40, 7749-7759 (2017)

30. Mohiuddine, S.A., Acar, T., Alotaibi, A.: Durrmeyer type $(p, q)$-Baskakov operators preserving linear functions. J. Math. Inequal. 12, 961-973 (2018)

31. Mohiuddine, S.A., Alamri, B.A.S.: Generalization of equi-statistical convergence via weighted lacunary sequence with associated Korovkin and Voronovskaya type approximation theorems. Rev. R. Acad. Cienc. Exactas Fís. Nat., Ser. A Mat. 113(3), 1955-1973 (2019)

32. Mohiuddine, S.A., Ōzger, F.: Approximation of functions by Stancu variant of Bernstein-Kantorovich operators based on shape parameter $\alpha$. Rev. R. Acad. Cienc. Exactas Fís. Nat., Ser. A Mat. 114, 70 (2020)

33. Mursaleen, M., Ansari, K.J., Khan, A.: On (p, q)-analogue of Bernstein operators. Appl. Math. Comput. 266, 874-882 (2015)

34. Mursaleen, M., Ansari, K.J., Khan, A.: Some approximation results by $(p, q)$-analogue of Bernstein-Stancu operators. Appl. Math. Comput. 264, 392-402 (2015)

35. Mursaleen, M., Naaz, A., Khan, A.: Improved approximation and error estimations by King type (p, q)-Szász-Mirakjan-Kantorovich operators. Appl. Math. Comput. 348, 175-185 (2019)

36. Mursaleen, M., Nasiruzzaman, M.: Approximation of modified Jakimovski-Leviatan-beta type operators. Constr. Math Anal. 1(2), 88-98 (2018)

37. Mursaleen, M., Nasiruzzaman, Md., Alotaibi, A.: On modified Dunkl generalization of Szász operators via q-calculus. J. Inequal. Appl. 2017, 38 (2017)

38. Nasiruzzaman, M., Mukheimer, A., Mursaleen, M.: A Dunkl-type generalization of Szász-Kantorovich operators via post quantum calculus. Symmetry 11(2), 232 (2019)

39. Nasiruzzaman, M., Mukheimer, A., Mursaleen, M.: Approximation results on Dunkl generalization of Phillips operators via $q$-calculus. Adv. Differ. Equ. 2019, 244 (2019)

40. Ōzger, F., Srivastava, H.M., Mohiuddine, S.A.: Approximation of functions by a new class of generalized Bernstein-Schurer operators. Rev. R. Acad. Cienc. Exactas Fís. Nat., Ser. A Mat. 114, 173 (2020)

41. Peetre, J.: A theory of interpolation of normed spaces. Noteas de Mathematica, Instituto de Mathemática Pura e Applicada, Conselho Nacional de Pesquidas, Rio de Janeiro 39 (1968)

42. Phillips, G.M.: Bernstein polynomials based on the $q$-integers, The heritage of P.L. Chebyshev, A Festschrift in honor of the 70th-birthday of Professor T.J. Rivlin. Ann. Numer. Math. 4, 511-518 (1997)

43. Rao, N., Wafi, A.: (p, q)-Bivariate-Bernstein-Chlodowsky operators. Filomat 32(2), 369-378 (2018)

44. Rao, N., Wafi, A.: Bivariate-Schurer-Stancu operators based on (p, q)-integers. Filomat 32(4), 1251-1258 (2018)

45. Rao, N., Wafi, A., Acu, A.M.: q-Szász-Durrmeyer type operators based on Dunkl analogue. Complex Anal. Oper. Theory 13(3), 915-934 (2019)

46. Sucu, S.: Dunkl analogue of Szász operators. Appl. Math. Comput. 244, 42-48 (2014)

47. Szász, O.: Generalization of S. Bernstein's polynomials to the infinite interval. J. Res. Natl. Bur. Stand. 45, 239-245 (1950)

48. Wafi, A., Rao Deepmala, N.: Approximation properties of $(p, q)$-variant of Stancu-Schurer operators. Bol. Soc. Parana. Mat. 37(4), 137-150 (2019) 